\title{
Assessment of Potential Risk due to Accidental Melting of Scrap Metal Containing Depleted Uranium Using a Computational Method
}

\author{
Elsayeda Farid Salem ${ }^{1}$, Mohamed Abdelati², Kamel Mohamed El Kourghly ${ }^{2}$ \\ ${ }^{1}$ Nuclear Law and Nuclear Licensee Department, Egypt Nuclear and Radiological Regulatory Authority, Cairo, Egypt \\ ${ }^{2}$ Nuclear Safeguards and Physical Protection Department, Egypt Nuclear and Radiological Regulatory Authority, Cairo, Egypt
}

Email address:

sayeda_f@yahoo.com (E. F. Salem), aty1611983@yahoo.com (M. Abdelati), eaea_nsncrc@yahoo.com (K. M. El Koughly)

\section{To cite this article:}

Elsayeda Farid Salem, Mohamed Abdelati, Kamel Mohamed El Kourghly. Assessment of Potential Risk due to Accidental Melting of Scrap Metal Containing Depleted Uranium Using a Computational Method. American Journal of Environmental Science and Engineering. Vol. 3, No. 3, 2019, pp. 60-65. doi: 10.11648/j.ajese.20190303.13

Received: January 26, 2019; Accepted: April 3, 2019; Published: October 31, 2019

\begin{abstract}
Depleted uranium (DU) has a beneficial use, such as ballast in aircraft and radiation shielding. Due to the chemical and radiological toxicity it may have adverse consequences to human health, particularly if it enters the body through inhalation, ingestion or wounding. One significant problem area, when working with DU, comes from finely divided airborne particles, which can result from some manufacturing operations such as machining and grinding. In this study RESRADRecycle computer code is used to estimate the exposure of workers and public to the recycling of scrap metal including depleted uranium and to evaluate the risk. Two general types of exposure scenarios have been incorporated into RESRADRecycle. The First scenario (worker scenario), evaluates worker's doses during the recycled material process. The second scenario (product scenario), determines public dose and risk from the use or exposure to products made of contaminated scrap metal. The obtained results indicate that the slag worker exposed to the highest dose and risk. In addition, the produced products cause a public hazard. Therefore, strengthen the nuclear safety and security regulations to this material type is mandatory. Spreading safety, security and safeguard culture is requisite to reduce the hazards of the radioactive materials.
\end{abstract}

Keywords: Depleted Uranium, RESRAD-Recycle Computer Code, Dose, Recycled Radioactive Materials

\section{Introduction}

Depleted uranium differs from natural uranium by having most of its U-235 and U-234 isotopes removed in the enrichment proses for nuclear fuel or weapons. However, its chemical and biological behavior is virtually identical to that of natural uranium [1]. Depleted uranium (DU) has a beneficial use, such as ballast in aircraft and radiation shielding. Due to the chemical and radiological toxicity it may have adverse consequences to human health, particularly if it enters the body through inhalation, ingestion or wounding. One significant problem area, when working with DU, comes from finely divided airborne particles, which can result from some manufacturing operations such as machining and grinding. Recently, depleted uranium (DU) use in peaceful applications are increased widely, since it is nearly twice as dense as lead. It can be used as counterweights or ballast in aircraft, parts of shielding material in a radiotherapy machine as well as container for the transport of radioactive materials [2]. Depleted counterweights used in the aircraft are triangular prisms manufactured from cast depleted uranium (DU). The counterweights are flash coated and painted with a primer to reduce surface oxidation [3]. In a typical sample of depleted uranium, most of the weight $(99.8 \%)$ consists of atoms of U238. about $(0.2 \%)$ of the weight consists of atoms of $U-235$, and a very small amount $(0.0006 \%$ by weight $)$ is $\mathrm{U}-234$. Thus, ${ }^{238} \mathrm{U}$ isotope in DU is the radiological concern. Radioactivity emitted from uranium isotopes consists of alpha particles and gamma rays. Activity of DU is 14.80 $\mathrm{Bq} / \mathrm{mg}$ which is about $58 \%$ of the activity of natural uranium. DU is 3 million times less radioactive than ${ }^{226} \mathrm{Ra}$ (still found in many old luminous clocks and watches) and 10 million times less radioactive than ${ }^{241} \mathrm{Am}$, which is found in 
commercial fire detectors [4-5]. The radiological hazards of any radioactive material are proportional to the amount of radioactivity present. The various uranium isotopes, and mixtures of those isotopes, can be characterized by their "specific activity", defined as the amount of radioactivity (in Curies) per unit of mass (in gram). Radionuclides with longer half-lives have smaller specific activities [6]. Because of U238 has very long half-life, the effects of decay on its concentration is not significant for short time periods [7]. The ${ }^{238} \mathrm{u}$ is an alpha emitter and as such poses no real risk to any of the sensitive tissues of the body including the lenses of the eyes a t a depth of $3 \mathrm{~mm}$ and the basal layer of the epidermis at $70 \mathrm{pm}$. This nonpenetrating radiation interacts only with the outer dermal layer and, at scenario atmospheric levels, would have no significant effect. The beta radiation in the plume could pose a hazard to uncovered skin, and gamma radiation would contribute to the whole-body dose. However, these dose contributions are expected to be insignificant [8].

Therefore, exposure effect of DU is mainly the result of its ingestion, inhalation and dermal contact [9]. Entry of uranium into the body thus results in a combined chemical/radiation exposure. In the work place or the environment, the radiological hazards from DU are primarily due to alpha particle emission. This means that the internal radiation dose from ingestion or inhalation of uranium compounds is the limiting hazard under almost all circumstances [10]. Beta and gamma components are only the DU contribute to external dose where the affected organ is the skin, External exposure to DU occurs when fragments are picked up $[11,12]$. Potentially depleted uranium has both chemical and radiological toxicity with the two important target organs being the kidneys and the lungs. Health consequences are determined by the physical and chemical nature of the depleted uranium to which an individual is exposed, and to the level and duration of exposure. Ingestion of DU is not considered the major exposure pathway [13]. Inhalation of dust is considered the major pathway for DU exposure. It is essential to provide machine ventilation, area ventilation and special filtering equipment to protect workers from radioactive dust and particles that could be inhaled or ingested [14]. Like any radioactive material, there is a risk of developing cancer from exposure to radiation emitted by natural and depleted uranium. The annual dose limit set by the IAEA for a member of the public is $1 \mathrm{mSv}$, while the corresponding limit for a radiation worker is $20 \mathrm{mSv}$. The additional risk of fatal cancer associated with a dose of 1 $\mathrm{mSv}$ is assumed to be about 1 in 20,000. This small increase in lifetime risk should be considered in light of the risk of 1 in 5 that everyone has of developing a fatal cancer. It must also be noted that cancer may not become apparent until many years after exposure to a radioactive material [15].

\section{Material and Method}

The RESRAD-RECYCLE computer code is a pathway analysis tool designed to calculate potential radiation doses and risks resulting from recycling of radioactive scrap metal and the reuse of surface-contaminated material and equipment RESRAD-RECYLE is a member of the RESRAD family of codes developed by Argonne National Laboratory USA department of energy.

I Description of the scenarios

Two general types of exposure scenarios have been incorporated into RESRAD-RECYCLE:

1 Worker scenarios:

For evaluating the dose and risk to workers who process recycled materials

The worker scenarios model potential exposures are associated with:

a) The transport of radioactive scrap metal from the place of origin to the smelter (step 1, scrap delivery),

b) The smelting process and manufacture of metal ingots for industrial products (step 2, scrap smelting),

c) Transport of metal ingots to product fabrication plants (step3 ingot delivery),

d) Product fabrication (step 4, initial and final fabrications)

2 End-use product scenarios:

For evaluating the dose and risk to persons using or otherwise being exposed to products made of recycled radioactive materials [16].

The Consumer scenarios are including:

a) public products (pavement, bridges and buildings),

b) surface contaminated reuse products (tools and contaminated buildings)

II Model input parameters:

A postulated accident Depleted uranium with weight of $150 \mathrm{Kg}$ was missing and that managed to pass through the scrap metal recycling plant. The input model parameters are illustrated in Table 1.

Table 1. Input Parameters of Selected Scenarios.

\begin{tabular}{|c|c|c|c|c|c|c|c|c|}
\hline Scenario & & $\begin{array}{l}\text { Dilution } \\
\text { Fraction }\end{array}$ & Exp Time hr & $\begin{array}{l}\text { Dust Load } \\
\mathrm{g} / \mathrm{m}^{3}\end{array}$ & Resp Fract & Inh Rate $\mathrm{m}^{3} / \mathrm{hr}$ & $\begin{array}{l}\text { Inh Protec } \\
\text { Factor }\end{array}$ & $\begin{array}{l}\text { Ing Rate } \\
\text { g/hr }\end{array}$ \\
\hline Scrap Delivery: & Scrap Cutter & $1.00 \mathrm{E}+00$ & $1.20 \mathrm{E}+01$ & $5.00 \mathrm{E}-04$ & $1.00 \mathrm{E}-01$ & $1.20 \mathrm{E}+00$ & $1.00 \mathrm{E}+00$ & $6.25 \mathrm{E}-03$ \\
\hline Scrap Delivery: & Scrap Loader & $1.00 \mathrm{E}+00$ & $4.00 \mathrm{E}+00$ & $5.00 \mathrm{E}-04$ & $1.00 \mathrm{E}-01$ & $1.20 \mathrm{E}+00$ & $1.00 \mathrm{E}+00$ & $6.25 \mathrm{E}-03$ \\
\hline Scrap Delivery: & Scrap Truck Driver & $1.00 \mathrm{E}+00$ & $4.00 \mathrm{E}+00$ & $0.00 \mathrm{E}+00$ & $1.00 \mathrm{E}-01$ & $0.00 \mathrm{E}+00$ & $1.00 \mathrm{E}+00$ & $0.00 \mathrm{E}+00$ \\
\hline Scrap Smelting: & Scrap Processor & $1.00 \mathrm{E}+00$ & $1.20 \mathrm{E}+01$ & $1.00 \mathrm{E}-04$ & $1.00 \mathrm{E}-01$ & $1.20 \mathrm{E}+00$ & $1.00 \mathrm{E}+00$ & $6.25 \mathrm{E}-03$ \\
\hline Scrap Smelting: & Smelter Yard Worker & $1.00 \mathrm{E}+00$ & $8.00 \mathrm{E}+01$ & $1.00 \mathrm{E}-04$ & $1.00 \mathrm{E}-01$ & $1.20 \mathrm{E}+00$ & $1.00 \mathrm{E}+00$ & $6.25 \mathrm{E}-03$ \\
\hline Scrap Smelting: & Smelter Loader & $1.00 \mathrm{E}+00$ & $4.00 \mathrm{E}+00$ & $1.00 \mathrm{E}-03$ & $1.00 \mathrm{E}-01$ & $1.20 \mathrm{E}+00$ & $1.00 \mathrm{E}+00$ & $6.25 \mathrm{E}-03$ \\
\hline Scrap Smelting: & Furnance Operator & $1.00 \mathrm{E}+00$ & $5.00 \mathrm{E}+00$ & $1.00 \mathrm{E}-03$ & $1.00 \mathrm{E}-01$ & $1.20 \mathrm{E}+00$ & $1.00 \mathrm{E}+00$ & $6.25 \mathrm{E}-03$ \\
\hline Scrap Smelting: & Baghouse Processor & $1.00 \mathrm{E}+00$ & $1.00 \mathrm{E}+00$ & $1.00 \mathrm{E}-03$ & $1.00 \mathrm{E}-01$ & $1.20 \mathrm{E}+00$ & $1.00 \mathrm{E}+00$ & $6.25 \mathrm{E}-03$ \\
\hline
\end{tabular}




\begin{tabular}{|c|c|c|c|c|c|c|c|c|}
\hline Scenario & & $\begin{array}{l}\text { Dilution } \\
\text { Fraction } \\
\end{array}$ & Exp Time hr & $\begin{array}{l}\text { Dust Load } \\
\mathrm{g} / \mathrm{m}^{3}\end{array}$ & Resp Fract & Inh Rate $\mathbf{m}^{3} / \mathbf{h r}$ & $\begin{array}{l}\text { Inh Protec } \\
\text { Factor }\end{array}$ & $\begin{array}{l}\text { Ing Rate } \\
\mathrm{g} / \mathrm{hr}\end{array}$ \\
\hline Scrap Smelting: & Refinery Worker & $1.00 \mathrm{E}+00$ & $5.00 \mathrm{E}+00$ & $1.00 \mathrm{E}-03$ & $1.00 \mathrm{E}-01$ & $1.20 \mathrm{E}+00$ & $1.00 \mathrm{E}+00$ & $6.25 \mathrm{E}-03$ \\
\hline Scrap Smelting: & Ingot Caster & $1.00 \mathrm{E}+00$ & $2.50 \mathrm{E}+00$ & $1.00 \mathrm{E}-03$ & $1.00 \mathrm{E}-01$ & $1.20 \mathrm{E}+00$ & $1.00 \mathrm{E}+00$ & $6.25 \mathrm{E}-03$ \\
\hline Scrap Smelting: & Small Objects Caster & $1.00 \mathrm{E}+00$ & $5.00 \mathrm{E}+01$ & $1.00 \mathrm{E}-03$ & $1.00 \mathrm{E}-01$ & $1.20 \mathrm{E}+00$ & $1.00 \mathrm{E}+00$ & $6.25 \mathrm{E}-03$ \\
\hline Scrap Smelting: & Slag Worker & $1.00 \mathrm{E}+00$ & $2.50 \mathrm{E}+01$ & $1.00 \mathrm{E}-03$ & $1.00 \mathrm{E}-01$ & $1.20 \mathrm{E}+00$ & $1.00 \mathrm{E}+00$ & $6.25 \mathrm{E}-03$ \\
\hline Ingot Delivery: & Ingot Loader & $1.00 \mathrm{E}+00$ & $2.00 \mathrm{E}+00$ & $0.00 \mathrm{E}+00$ & $1.00 \mathrm{E}-01$ & $0.00 \mathrm{E}+00$ & $1.00 \mathrm{E}+00$ & $0.00 \mathrm{E}+00$ \\
\hline Ingot Delivery: & Ingot Truck Driver & $1.00 \mathrm{E}+00$ & $5.00 \mathrm{E}+00$ & $0.00 \mathrm{E}+00$ & $1.00 \mathrm{E}-01$ & $0.00 \mathrm{E}+00$ & $1.00 \mathrm{E}+00$ & $0.00 \mathrm{E}+00$ \\
\hline Initial Fabrication: & Storage Yard Worker & $1.00 \mathrm{E}+00$ & $4.00 \mathrm{E}+01$ & $0.00 \mathrm{E}+00$ & $1.00 \mathrm{E}-01$ & $0.00 \mathrm{E}+00$ & $1.00 \mathrm{E}+00$ & $0.00 \mathrm{E}+00$ \\
\hline Initial Fabrication: & Sheet Maker & $1.00 \mathrm{E}+00$ & $1.00 \mathrm{E}+00$ & $1.00 \mathrm{E}-04$ & $1.00 \mathrm{E}-01$ & $1.20 \mathrm{E}+00$ & $1.00 \mathrm{E}+00$ & $6.25 \mathrm{E}-03$ \\
\hline Initial Fabrication: & Coil Maker & $1.00 \mathrm{E}+00$ & $1.00 \mathrm{E}+00$ & $1.00 \mathrm{E}-04$ & $1.00 \mathrm{E}-01$ & $1.20 \mathrm{E}+00$ & $1.00 \mathrm{E}+00$ & $6.25 \mathrm{E}-03$ \\
\hline Final Fabrication: & Sheet Handler & $1.00 \mathrm{E}+00$ & $1.00 \mathrm{E}+00$ & $0.00 \mathrm{E}+00$ & $1.00 \mathrm{E}-01$ & $0.00 \mathrm{E}+00$ & $1.00 \mathrm{E}+00$ & $0.00 \mathrm{E}+00$ \\
\hline Final Fabrication: & Coil Handler & $1.00 \mathrm{E}+00$ & $8.00 \mathrm{E}+01$ & $0.00 \mathrm{E}+00$ & $1.00 \mathrm{E}-01$ & $0.00 \mathrm{E}+00$ & $1.00 \mathrm{E}+00$ & $0.00 \mathrm{E}+00$ \\
\hline Product Distribution: & Product Loader & $1.00 \mathrm{E}+00$ & $2.00 \mathrm{E}+01$ & $0.00 \mathrm{E}+00$ & $1.00 \mathrm{E}-01$ & $0.00 \mathrm{E}+00$ & $1.00 \mathrm{E}+00$ & $0.00 \mathrm{E}+00$ \\
\hline Product Distribution: & Product Truck Driver & $1.00 \mathrm{E}+00$ & $8.00 \mathrm{E}+00$ & $0.00 \mathrm{E}+00$ & $1.00 \mathrm{E}-01$ & $0.00 \mathrm{E}+00$ & $1.00 \mathrm{E}+00$ & $0.00 \mathrm{E}+00$ \\
\hline Product Distribution: & Sheet Assembler & $1.00 \mathrm{E}+00$ & $2.00 \mathrm{E}+01$ & $0.00 \mathrm{E}+00$ & $1.00 \mathrm{E}-01$ & $0.00 \mathrm{E}+00$ & $1.00 \mathrm{E}+00$ & $0.00 \mathrm{E}+00$ \\
\hline Product Distribution: & Warehouse Worker & $1.00 \mathrm{E}+00$ & $2.00 \mathrm{E}+03$ & $0.00 \mathrm{E}+00$ & $1.00 \mathrm{E}-01$ & $0.00 \mathrm{E}+00$ & $1.00 \mathrm{E}+00$ & $0.00 \mathrm{E}+00$ \\
\hline Consumer Product: & Parking Lot & $1.00 \mathrm{E}-02$ & $6.20 \mathrm{E}+01$ & $0.00 \mathrm{E}+00$ & $1.00 \mathrm{E}-01$ & $0.00 \mathrm{E}+00$ & $1.00 \mathrm{E}+00$ & $0.00 \mathrm{E}+00$ \\
\hline Consumer Product: & Room/Office & $1.00 \mathrm{E}+00$ & $2.00 \mathrm{E}+03$ & $0.00 \mathrm{E}+00$ & $1.00 \mathrm{E}-01$ & $0.00 \mathrm{E}+00$ & $1.00 \mathrm{E}+00$ & $0.00 \mathrm{E}+00$ \\
\hline Consumer Product: & Appliance & $1.00 \mathrm{E}+00$ & $7.30 \mathrm{E}+02$ & $0.00 \mathrm{E}+00$ & $1.00 \mathrm{E}-01$ & $0.00 \mathrm{E}+00$ & $1.00 \mathrm{E}+00$ & $0.00 \mathrm{E}+00$ \\
\hline Consumer Product: & Automobile & $1.00 \mathrm{E}+00$ & $7.30 \mathrm{E}+02$ & $0.00 \mathrm{E}+00$ & $1.00 \mathrm{E}-01$ & $0.00 \mathrm{E}+00$ & $1.00 \mathrm{E}+00$ & $0.00 \mathrm{E}+00$ \\
\hline Consumer Product: & Office Furniture & $1.00 \mathrm{E}+00$ & $2.00 \mathrm{E}+03$ & $0.00 \mathrm{E}+00$ & $1.00 \mathrm{E}-01$ & $0.00 \mathrm{E}+00$ & $1.00 \mathrm{E}+00$ & $0.00 \mathrm{E}+00$ \\
\hline Consumer Product: & Home Furniture & $1.00 \mathrm{E}+00$ & $3.65 \mathrm{E}+03$ & $0.00 \mathrm{E}+00$ & $1.00 \mathrm{E}-01$ & $0.00 \mathrm{E}+00$ & $1.00 \mathrm{E}+00$ & $0.00 \mathrm{E}+00$ \\
\hline Consumer Product: & Frying Pan & $1.00 \mathrm{E}+00$ & $1.80 \mathrm{E}+02$ & $0.00 \mathrm{E}+00$ & $1.00 \mathrm{E}-01$ & $0.00 \mathrm{E}+00$ & $1.00 \mathrm{E}+00$ & 4.12E-03 \\
\hline Public Product: & Pavement & $1.00 \mathrm{E}-02$ & $6.00 \mathrm{E}+00$ & $0.00 \mathrm{E}+00$ & $1.00 \mathrm{E}-01$ & $0.00 \mathrm{E}+00$ & $1.00 \mathrm{E}+00$ & $0.00 \mathrm{E}+00$ \\
\hline Public Product: & Building with Rebars & $1.00 \mathrm{E}+00$ & $2.00 \mathrm{E}+03$ & $0.00 \mathrm{E}+00$ & $1.00 \mathrm{E}-01$ & $0.00 \mathrm{E}+00$ & $1.00 \mathrm{E}+00$ & $0.00 \mathrm{E}+00$ \\
\hline Public Product: & Bridge & $1.00 \mathrm{E}+00$ & $1.00 \mathrm{E}+00$ & $0.00 \mathrm{E}+00$ & $1.00 \mathrm{E}-01$ & $0.00 \mathrm{E}+00$ & $1.00 \mathrm{E}+00$ & $0.00 \mathrm{E}+00$ \\
\hline Controlled Products: & Shield Block & $1.00 \mathrm{E}+00$ & $1.00 \mathrm{E}+00$ & $0.00 \mathrm{E}+00$ & $1.00 \mathrm{E}-01$ & $0.00 \mathrm{E}+00$ & $1.00 \mathrm{E}+00$ & $0.00 \mathrm{E}+00$ \\
\hline Controlled Products: & Radwaste Container & $1.00 \mathrm{E}+00$ & $1.00 \mathrm{E}+00$ & $0.00 \mathrm{E}+00$ & $1.00 \mathrm{E}-01$ & $0.00 \mathrm{E}+00$ & $1.00 \mathrm{E}+00$ & $0.00 \mathrm{E}+00$ \\
\hline Reuse Product: & Tool Reuse & $1.00 \mathrm{E}-02$ & $2.00 \mathrm{E}+03$ & $1.00 \mathrm{E}-06$ & $1.00 \mathrm{E}-01$ & $1.20 \mathrm{E}+00$ & $1.00 \mathrm{E}+00$ & $1.00 \mathrm{E}-04$ \\
\hline Reuse Product: & Building Reuse & $1.00 \mathrm{E}-02$ & $2.00 \mathrm{E}+03$ & $1.00 \mathrm{E}-06$ & $1.00 \mathrm{E}-01$ & $1.20 \mathrm{E}+00$ & $1.00 \mathrm{E}+00$ & $1.00 \mathrm{E}-04$ \\
\hline Scrap Transportation: & Public Exposure & $1.00 \mathrm{E}+00$ & $1.00 \mathrm{E}+00$ & $0.00 \mathrm{E}+00$ & $1.00 \mathrm{E}-01$ & $0.00 \mathrm{E}+00$ & $1.00 \mathrm{E}+00$ & $0.00 \mathrm{E}+00$ \\
\hline
\end{tabular}

Note: For surface reuse scenarios, the dust load is the emission rate in $(1 / \mathrm{hr})$, the ingestion rate is in $\left(\mathrm{m}^{2} / \mathrm{hr}\right)$ and the dilution represents a surface transfer factor applied to inhalation and ingestion doses only.

Theoretical calculation for effective dose:

The estimated values of the external effective doses by the RESRAD - Recycle Code in this study results validated with the calculated values using the equation (1).

$$
\begin{aligned}
& \mathrm{D}= \frac{F_{7} A}{X^{2}} \\
& \text { Eext }=\frac{\mathrm{ACF} F_{6} T \mathrm{e}}{X^{2}}
\end{aligned}
$$

Where:

D: Dose rat (mSvlh)

$\mathrm{E}_{\text {ext }}=$ Effective dose from a point source $[\mathrm{mSv}]$

$\mathrm{A}=$ Source activity $[\mathrm{kBq}]$

$\mathrm{T}_{\mathrm{e}}=$ Exposure duration $[\mathrm{h}]$

$\mathrm{CF}_{6}=$ Conversion factor $2.3 \times 10^{-1}[(\mathrm{mSv} / \mathrm{h}) /(\mathrm{kBq})]$ (For natural and depleted uranium it is assumed all of the release is U-238) [17]

F7: Conversion factor

$\mathrm{X}=$ Distance from the point source $[\mathrm{m}]$

\section{Result and Discussion}

The output data effective doses for working and scrap delivery scenarios (including scrap cutter, scrap loader, scrap truck driver) for the total three uranium isotopes in DU of scrap metal are presented in Table 2. Also, smelting work scenarios include scrap processor, refinery worker, and slag worker, as illustrated in Table 3. The effective doses (ingestion, inhalation, external, collective, cumulative) for scrap working scenario are presented in Figures 1, 2 and 3, respectively. It is clear that at the scrap delivery stage the ingestion, inhalation and external dose are higher than code of conduct of IAEA by a factor of 6 , where incidental ingestion of particulates attaches to the hands during cutting process. Workers inhale high dose in the scrap cutter and scrap loader and are exposed to a high external dose during truck driver. From Figure 2 it is obvious that for the smelter yard worker the ingestion dose and the inhalation dose were about $6.5 \mu \mathrm{Sv}$ and $5.4 \mu \mathrm{Sv}$ respectively, which are about six times Code of Conduct IAEA limit [18]. The worker be inside the vicinity of smelting facility are exposed to radioactive air particulates. The inhalation dose for the smelter loader worker and furnance operator were about $27 \mu \mathrm{Sv}$ and $37 \mu \mathrm{Sv}$ respectively. In Figure 3 the highest dose was for slag worker where the inhalation dose was about 0.2 $\mathrm{mSv}$ takes in 25 hours. Slag worker was also exposed to external of dose about $0.35 \mathrm{mSv}$. From the above results it turns out that slag worker is exposed to the highest dose in worker scenario. Table 4 and Figure 4 represent the fifths stages of the consumer scenario. It is noted that tool reuse and building reuse stages give high dose for ingestion, inhalation and external dose respectively, whereas public affected external dose is about $0.28 \mathrm{mSv}$ for 6 hours exposure 
when the slag is used in pavement. NRC and USDOE performance standards for both operational periods and the long-term protection of human health and the environment are fundamentally based on maximum allowable radiation dose levels, setting a maximum annual radiation dose to the public of $0.25 \mathrm{mSv}$ [19].
The most effective dose was in the scrap product public scenario (building Reuse product) for ingestion and external of about $1.6 \mathrm{mSv}$ and $1.4 \mathrm{mSv}$ per year respectively. These results are within the range of agreement if they are compared with the results obtained using equation (2).

Table 2. Effective dose equivalents ( $\mu$ Sv) scrap delivery (working scenario).

\begin{tabular}{|c|c|c|c|c|}
\hline \multirow{2}{*}{ Scenario } & & \multicolumn{2}{|c|}{ Effective dose Equivalents $(\mu \mathrm{Sv})$} & \multirow{2}{*}{ No of exposure people } \\
\hline & & DU & IAEA & \\
\hline \multirow{5}{*}{ Scrap Cutter scenario } & Ingestion & $1.04 \mathrm{E}+02$ & $1.8 \mathrm{E}-1$ & \multirow{5}{*}{3} \\
\hline & Inhalation & $4.04 \mathrm{E}+02$ & 7.27E-1 & \\
\hline & External & $\mathrm{E}+01.661$ & $7.62 \mathrm{E}+0$ & \\
\hline & Collective & $1.53 \mathrm{E}-04$ & $2.75 \mathrm{E}-6$ & \\
\hline & Cumulative & $1.53 \mathrm{E}-04$ & $2.75 \mathrm{E}-6$ & \\
\hline \multirow{5}{*}{ Scrap loader } & Ingestion & $1.46 \mathrm{E}+01$ & $6.01 \mathrm{E}-2$ & \multirow{5}{*}{2} \\
\hline & Inhalation & $0.45 \mathrm{E}+02$ & $2.42 \mathrm{E}-1$ & \\
\hline & External & $2.28 \mathrm{E}+00$ & $1.08 \mathrm{E}-2$ & \\
\hline & Collective & $1.49 \mathrm{E}-04$ & $6.27 \mathrm{E}-07$ & \\
\hline & Cumulative & $1.49 \mathrm{E}-04$ & $6.27 \mathrm{E}+0$ & \\
\hline \multirow{3}{*}{ Truck drive } & External & $4.33 \mathrm{E}+00$ & $9.68 \mathrm{E}-03$ & \multirow{3}{*}{4} \\
\hline & Collective & $2.33 \mathrm{E}-05$ & 4.84E-08 & \\
\hline & cumulative & $2.33 \mathrm{E}-05$ & 4.84E-08 & \\
\hline \multirow{5}{*}{ Scrap processor } & Ingestion & $4.71 \mathrm{E}+01$ & $1.69 \mathrm{E}-01$ & \multirow{5}{*}{3} \\
\hline & Inhalation & $4.08 \mathrm{E}+01$ & $1.45 \mathrm{E}-01$ & \\
\hline & External & $9.72 \mathrm{E}+00$ & $7.69 \mathrm{E}-03$ & \\
\hline & Collective & $5.45 \mathrm{E}-05$ & $9.66 \mathrm{E}-07$ & \\
\hline & comulative & $5.45 \mathrm{E}-05$ & $9.66 \mathrm{E}-07$ & \\
\hline \multirow{5}{*}{ Smelter yard worker } & Ingestion & $6.47 \mathrm{E}+01$ & $1.13 \mathrm{E}+00$ & \multirow{5}{*}{10} \\
\hline & Inhalation & $5.39 \mathrm{E}+01$ & $9.7 \mathrm{E}-01$ & \\
\hline & External & $3.34 \mathrm{E}+00$ & $6.68 \mathrm{E}-02$ & \\
\hline & Collective & $1.22 \mathrm{E}-03$ & $2.16 \mathrm{E}-05$ & \\
\hline & comulative & $1.22 \mathrm{E}-03$ & $2.16 \mathrm{E}-05$ & \\
\hline \multirow{5}{*}{ Smelter loder } & Ingestion & $3.73 \mathrm{E}+00$ & $6.49 \mathrm{E}-02$ & \multirow{5}{*}{5} \\
\hline & Inhalation & $2.69 \mathrm{E}+01$ & $4.85 \mathrm{E}-01$ & \\
\hline & External & $1.29 \mathrm{E}+00$ & $1.28 \mathrm{E}-02$ & \\
\hline & Collective & $1.56 \mathrm{E}-04$ & $2.81 \mathrm{E}-06$ & \\
\hline & comulative & $1.56 \mathrm{E}-04$ & $2.81 \mathrm{E}-06$ & \\
\hline \multirow{5}{*}{ Furnance Operator } & Ingestion & $4.67 \mathrm{E}+00$ & $8.11 \mathrm{E}-02$ & \multirow{5}{*}{3} \\
\hline & Inhalation & $3.37 \mathrm{E}+01$ & $6.06 \mathrm{E}-01$ & \\
\hline & External & $2.25 \mathrm{E}+00$ & 4.61E-02 & \\
\hline & Collective & $1.22 \mathrm{E}-04$ & $2.2 \mathrm{E}-06$ & \\
\hline & Comulative & $1.22 \mathrm{E}-04$ & $2.2 \mathrm{E}-06$ & \\
\hline
\end{tabular}

Table 3. Effective dose equivalents ( $\mu$ Sv) scrap smelting (working scenario).

\begin{tabular}{|c|c|c|c|c|}
\hline \multirow{2}{*}{ Scenario } & & \multicolumn{2}{|c|}{ Effective dose Equivalents $(\mu \mathrm{Sv})$} & \multirow{2}{*}{ No of exposure people } \\
\hline & & DU & IAEA & \\
\hline \multirow{5}{*}{ Baghouse processor } & Ingestion & $9.33 \mathrm{E}+00$ & $1.62 \mathrm{E}-02$ & \multirow{5}{*}{1} \\
\hline & Inhalation & $6.73 \mathrm{E}+01$ & $1.21 \mathrm{E}-01$ & \\
\hline & External & $1.02 \mathrm{E}+00$ & $2.17 \mathrm{E}-03$ & \\
\hline & Collective & 7.77E-05 & $1.4 \mathrm{E}-07$ & \\
\hline & cumulative & 7.77E-05 & $1.4 \mathrm{E}-07$ & \\
\hline \multirow{5}{*}{ Refinery worker } & Ingestion & $4.67 \mathrm{E}+00$ & $8.11 \mathrm{E}-02$ & \multirow{5}{*}{3} \\
\hline & Inhalation & $3.37 \mathrm{E}+00$ & $6.06 \mathrm{E}-01$ & \\
\hline & External & $0.00 \mathrm{E}+00$ & $0.00 \mathrm{E}+00$ & \\
\hline & Collective & $1.15 \mathrm{E}-04$ & $2.06 \mathrm{E}-06$ & \\
\hline & Cumulative & $1.15 \mathrm{E}-04$ & $2.06 \mathrm{E}-06$ & \\
\hline \multirow{5}{*}{ Slag worker } & Ingestion & $2.31 \mathrm{E}+02$ & $4.02 \mathrm{E}+00$ & \multirow{5}{*}{1} \\
\hline & Inhalation & $1.67 \mathrm{E}+03$ & $3.00 \mathrm{E}+01$ & \\
\hline & External & $3.46 \mathrm{E}+02$ & $6.50 \mathrm{E}+00$ & \\
\hline & Collective & $2.21 \mathrm{E}-04$ & $4.05 \mathrm{E}-05$ & \\
\hline & Comulative & $2.21 \mathrm{E}-04$ & $4.05 \mathrm{E}-05$ & \\
\hline
\end{tabular}


Table 4. Effective dose equivalents ( $\mu$ Sv) scrap product (public scenario).

\begin{tabular}{lllll}
\hline \multirow{2}{*}{ Scenario } & & \multicolumn{2}{l}{ Effective dose Equivalents $(\boldsymbol{\mu S v})$} & IAEA \\
\cline { 3 - 4 } Public product pavement & DU & $1.45 \mathrm{E}-01$ & \\
& External & $2.83 \mathrm{E}+02$ & $1.47 \mathrm{E}-03$ & $820 \mathrm{E}+04$ \\
& Collective & $3.08 \mathrm{E}-01$ & $1.46 \mathrm{E}-02$ & \\
& cumulative & $3.07 \mathrm{E}+00$ & $4.43 \mathrm{E}+01$ & \\
& Ingestion & $2.55 \mathrm{E}+02$ & $3.18 \mathrm{E}-01$ & \\
Reuse product & Inhalation & $1.77 \mathrm{E}+01$ & $7.85 \mathrm{E}+00$ & \\
(Tool reuse) & External & $1.78 \mathrm{E}+03$ & $5.25 \mathrm{E}-05$ & \\
& Collective & $2.80 \mathrm{E}-03$ & $5.25 \mathrm{E}-04$ & \\
& cumulative & $2.79 \mathrm{E}-02$ & $4.46 \mathrm{E}+01$ & \\
& Ingestion & $1.57 \mathrm{E}+03$ & $1.83 \mathrm{E}+01$ & \\
Reuse product & Inhalation & $1.02 \mathrm{E}+03$ & $2.70 \mathrm{E}+01$ & \\
(Building reuse) & External & $1.42 \mathrm{E}+03$ & $3.60 \mathrm{E}-04$ & \\
& Collective & $1.75 \mathrm{E}-02$ & $1.08 \mathrm{E}-02$ \\
\hline
\end{tabular}

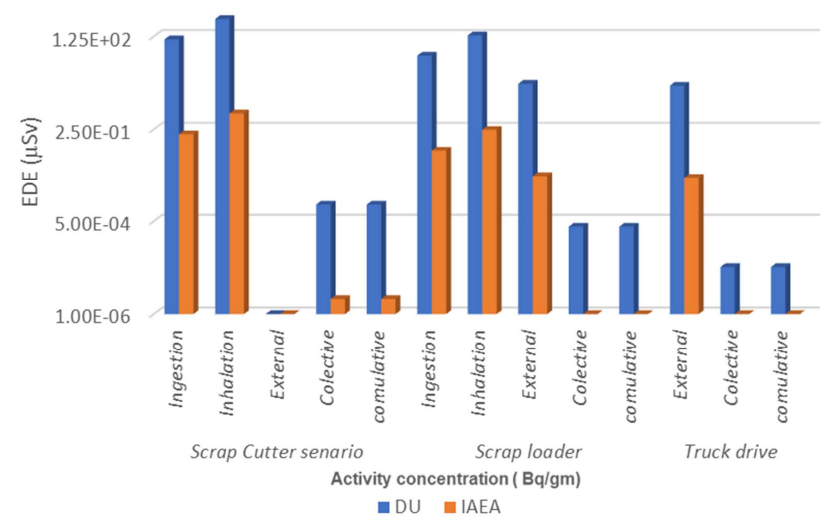

Figure 1. Scrap delivery include (cutter-loader-Truck driver).

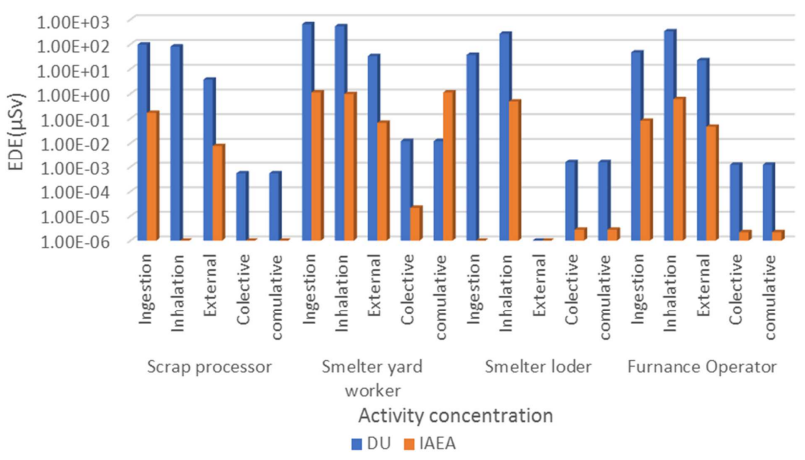

Figure 2. Smelting scenario for Scrap processor-smelting yard-smelting loader and furnance operator stages.

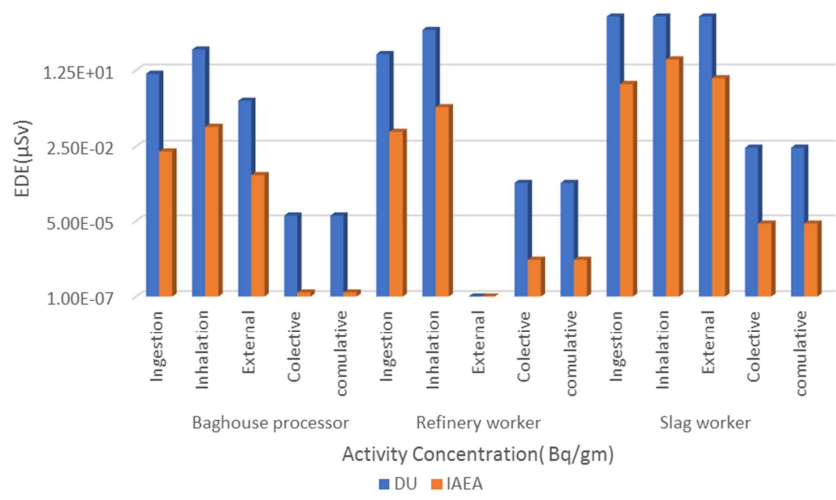

Figure 3. Smelting processor for Bughouse - refinery and slag worker stages.

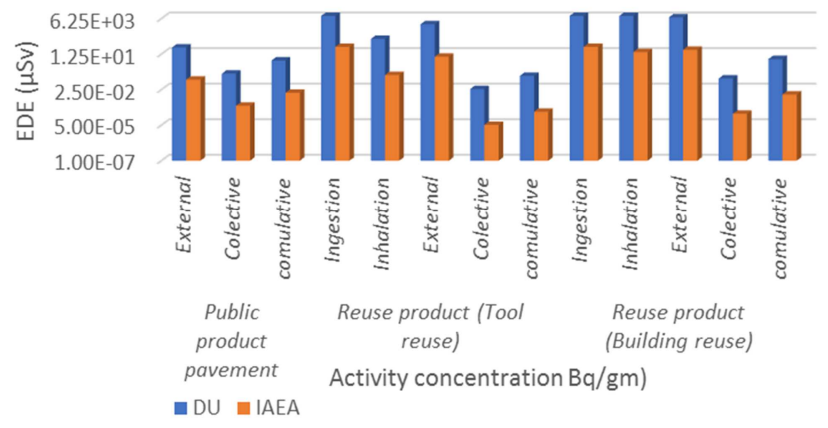

Figure 4. Consumer scenario include-pavement-tool reuse and building reuse.

\section{Conclusion}

Exposure effect of DU is mainly the result of its ingestion, inhalation where all uranium isotopes decay by alpha particles of various energies. Alpha particles have low penetrating power but deposit large amount of energy. Inhalation is the most likely route of intake of DU.

Public exposure to products incorporating radioactive scrap metal is most likely to result from external exposure. Melting radioactive source in metal recycle represent high hazard effect for slag worker, while for the public it is especially in tool reuse stage. Based on the zero-threshold linear dose response model, any absorbed dose of uranium is assumed to result in an increased risk of cancer. Since uranium tends to concentrate in specific locations in the body, the risk of cancer in the bone, liver, and blood (such as leukemia) may be increased [20].

Development of a worldwide recycling process will require further effort to determine the appropriate radioactivity limits for released materials to ensure protection of human health under possible conditions of exposure. It is important to provide the Scrap smelting places with ventilation machine, and special filtering equipment to protect workers from radioactive dust and particles that could be accidentally inhaled or ingested. All staff responsible for collecting, transporting and processing scrap metal should be provided with on-going training on the procedures in place in order to monitor for radiation and check for radioactive materials. Training should include how to recognize radiation 
symbols. Spreading safety, security and safeguard culture is requisite to reduce the hazards of radioactive materials.

\section{Acknowledgements}

The authors would like to thank Asmaa K. Abdien, of Quality Assurances Department, Nuclear and Radiological Regulatory Authority, Egypt for her contribution of the using the RESRAD-Recycle cod.

\section{References}

[1] Department of Protection of the Human Environment World Health Organization "Depleted uranium Sources, Exposure and Health Effects "Geneva, April 2001.

[2] International Atomic Energy Agency, "Depleted Uranium" Vienna, 1998-2017.

[3] Thomas D. Gallacher," Use of depleted uranium in aircraft" Radiation Safety Officer Senior Manager Corporate Radiation Health Protection Mail Stop 6Y-38, The Boeing Company September 8, 1994.

[4] A. Blaise, P. R. Danes, W. Burkart " Properties, use and health effects of depleted uranium (DU): a general overview" Journal of Environmental Radioactivity 64 (2003) 93-112.

[5] Eric Adijanto "Hazards of Depleted Uranium" Submitted as coursework for PH241, Stanford University, Winter 2012 March 21, 2012.

[6] T. J. Hartzler et.al. "Depleted Uranium Disposal Options Evaluation" EGG-MS-11297May 1994.

[7] J. Mishima M. A, Parkhurst R, Scherpelz D. E. "Potential Behavior of Depleted Uranium Penetrators under Shipping and Bulk Storage Accident Conditions" Hadlock March 1985.

[8] R. I. Scherpelz, J. Mishima and M. A. Parkhurst (Pacific Northwest Laboratory), Documented in a letter report, "Calculations of the Effects of Shipping and Bulk Storage
Accidents Involving Depleted Uranium Penetrators," to the U.S. Army in 1984.

[9] John R. Brodeur, P. E., L. E. G. Energy Sciences \& Engineering Kennewick, WAA Review and Comparison of Low-Level Radioactive Waste Disposal Facilities, September 2003.

[10] OECD Nuclear Energy Agency and the International Atomic Energy Agency Joint Report "Management of Depleted Uranium ", 2001.

[11] United States Nuclear Regulatory Commission "NRC Regulations (10 CFR) Part Index > $\$ 40.4$ Definitions ", Page Last Reviewed/Updated Tuesday, August 29, 2017.

[12] William E. Mumhi et.al." Assessment of recycling or disposal alternatives for radioactive scrap metal "Department of Energy and Chen; Argonne National Laboratory, 1993.

[13] (WHO, 2001; UNEP, 2001).

[14] Uranium Institute, "Depleted Uranium from Enrichment", London, November 1996.

[15] United States Nuclear Regulatory Commission (NRC) "Background Information on Depleted Uranium", August 09, 2017.

[16] USA department of energy "RESRAD- RECYCLE Computer Code Manual" Argon National laboratory, November 2000.

[17] IAEA "Generic procedures for assessment and response during a radiological emergency"TECDOC-1162, 2000, ISSN 1011-4289.

[18] IAEA General Safety Requirements Part 3 No. GSR Part 3 "Radiation Protection and Safety of Radiation Sources: International Basic Safety Standards" VIENNA, 2014.

[19] John R. Brodeur, P. E., L. E. G “A Review and Comparison of Low-Level Radioactive Waste Disposal facilities" Energy Sciences \& Engineering Kennewick, WA, September, 2003.

[20] U.S. Environmental Protection Agency "Depleted Uranium Technical Brief", EPA 402-R-06-011 Project Officer Brian Littleton December 2006. 